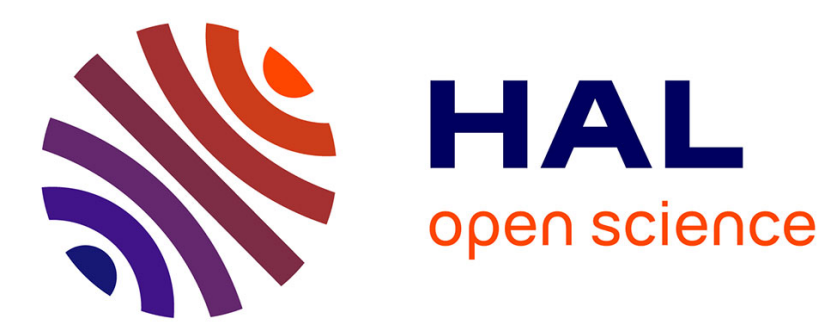

\title{
Experimental Evidence of Rotational Elastic Waves in Granular Phononic Crystals
}

\author{
Aurélien Merkel, Vincent Tournat, Vitali Goussev
}

\section{To cite this version:}

Aurélien Merkel, Vincent Tournat, Vitali Goussev. Experimental Evidence of Rotational Elastic Waves in Granular Phononic Crystals. Physical Review Letters, 2011, 107 (22), 10.1103/PhysRevLett.107.225502 . hal-01813224

\section{HAL Id: hal-01813224 \\ https://hal-univ-lemans.archives-ouvertes.fr/hal-01813224}

Submitted on 12 Jun 2018

HAL is a multi-disciplinary open access archive for the deposit and dissemination of scientific research documents, whether they are published or not. The documents may come from teaching and research institutions in France or abroad, or from public or private research centers.
L'archive ouverte pluridisciplinaire $\mathbf{H A L}$, est destinée au dépôt et à la diffusion de documents scientifiques de niveau recherche, publiés ou non, émanant des établissements d'enseignement et de recherche français ou étrangers, des laboratoires publics ou privés. 


\title{
Experimental Evidence of Rotational Elastic Waves in Granular Phononic Crystals
}

\author{
A. Merkel* and V. Tournat \\ LAUM, CNRS, Université du Maine, Av. O. Messiaen, 72085 Le Mans, France
}

V. Gusev

LPEC UMR-CNRS 6087, Université du Maine, Av. O. Messiaen, 72085 Le Mans, France

(Received 22 August 2011; published 21 November 2011)

\begin{abstract}
A generalized theory of elasticity, taking into account the rotational degrees of freedom of point bodies constituting a continuum, was proposed at the beginning of the twentieth century by the Cosserat brothers. We report the experimental observation of coupled rotational-translational modes in a noncohesive granular phononic crystal. While absent in the classical theory of elasticity, these elastic wave modes are predicted by the Cosserat theory. However the Cosserat theory fails to predict correctly the dispersion of the elastic modes in granular crystals even in the long-wavelength limit.
\end{abstract}

DOI: 10.1103/PhysRevLett.107.225502

PACS numbers: 62.20.D-, 45.70. $-\mathrm{n}, 62.30 .+\mathrm{d}$

One hundred years ago, the Cosserat brothers developed a continuum elasticity theory accounting for the rotational degrees of freedom of point bodies (infinitesimal particles) constituting deformable solids [1]. Currently, this theory is known as the Cosserat theory, and the related and advanced theories are known as theories of Cosserat continuum or as theories of micropolar continuum [2]. In the Cosserat theory, each material point has 6 degrees of freedom, three of which correspond to the translations as in the classical theory of elasticity, and the three others correspond to rotations. The stress tensor is asymmetric and an additional couple-stress tensor is introduced, which plays the same role for torques as the stress tensor plays for forces. The theory predicts a contribution of rotations to the dispersion of the shear elastic wave velocity as well as the existence of additional rotational wave modes [3]. For the description of an isotropic medium, the classical elasticity requires the knowledge of the elastic constants $\lambda$ and $\mu$ (the Lamé constants) and the material density $\rho$. The Cosserat theory requires four additional elastic constants $\alpha, \varepsilon, \gamma, \beta$, describing the microstructure, and the density of the moment of inertia $J[2,4]$.

However, even 100 years after the Cosserats, the experimental evidence of the Cosserat effects are rare, difficult to obtain, and subject to criticism $[2,3,5]$. The additional rotational mode resonances expected in the elastic vibration of macroscopic micropolar bodies [6] have never been observed. The determination and calibration of the additional Cosserat elastic constants from static or quasisatic experiments is delicate. In our opinion, the most crucial observation providing convincing evidence of the effects of the rotational degrees of freedom, the milestone of the Cosserat theory, would be the observation of the propagation of rotational waves in elastically micro-inhomogeneous media. The only cited observation [2,3] of rotational waves in a specimen composed of metallic particles randomly distributed in an epoxy polymer matrix [7] is not convincing [8]. The rotational waves in the Cosserat continuum theory is often considered a mathematical possibility, but proving their actual existence by experiments constitutes a serious endeavor [9].

Below, we demonstrate that important advantages for testing the fundamentals of the Cosserat theory can be gained through conducting the experiments in noncohesive phononic granular crystals which are not saturated by liquids. In noncohesive granular media, the macroscopic dynamical behavior depends on the peculiar shear and normal contact interactions between the spheres at the microscopic level, which are well described by the HertzMindlin theory of contact $[10,11]$. According to this theory, in the first aproximation, a weakly precompressed granular crystal, where the diameters $d$ of the contacts between the beads are much smaller than the diameter $a$ of the beads $(d / a \ll 1)$, can be reduced to a mass-spring structure. Since the size of the grains is comparable to the distance between neighbors, the rotational degrees of freedom of the beads must be taken into account in order to accurately describe the dynamics of the granular media $[12,13]$. However, while the bending rigidity of the contacts [14] and their spin (torsional) rigidity generally contribute to the effects of the rotation of the beads $[9,15]$, their contribution is not needed here. The moment $[1,3]$ applied to the beads due to these two rigidities is about $(a / d)^{2} \gg 1$ times smaller than the moment applied to the beads due to the shear rigidity. In the Cosserat continuum, the absence of torsional and bending rigidities corresponds to negligible nonclassical elastic constants $\varepsilon, \gamma$, and $\beta$ and to the absence of the momentum stresses acting on the contacts between the beads. Thus, the only nonclassical elastic constant expected to play an important role in noncohesive granular crystals is $\alpha$, which introduces asymmetry of the stress tensor. These circumstances provide important simplifications in identifying the contribution of rotational degrees of freedom to elastic wave motion 
in the noncohesive air-filled granular crystals in comparison with cohesive crystals or crystals infiltrated with other media forming the matrix around the beads [16-21], where the couple-stress tensor is not negligible. Here, we report the experimental observations of rotational elastic waves. A hexagonal close-packed (hcp) structure of identical elastic beads is a vertical stacking of hexagonal layers $A$ and $B$, which are in the closest position relative to each others, in an $A B A B \ldots$ sequence. In such packing, the theory predicts the existence of translational modes, rotational modes, and coupled rotational and translational modes [13]. The dispersion relations and the allowed frequency bands for the propagation of different elastic modes can be analytically predicted in the considered periodic granular media. This facilitates the identification of the various propagating modes. At frequencies much lower than the rotational frequency bands, only the dispersionless longitudinal and shear acoustic waves have been monitored in the classical experiments [10]. At frequencies much higher than the rotational frequency bands, the elastic signals related to torsional and spheroidal resonances of the individual beads and Rayleigh wave propagation on the surface of the individual beads have been detected [22,23]. In our experiments, due to the relatively weak static loading applied to the crystal, the rotational wave frequencies are about 10 times lower than the lowest vibrational resonances of the individual beads. Thus, it is possible to observe the effects of wave dispersion and forbidden zones due to the medium periodicity without interference from scattering phenomena due to the individual resonances of the beads [24].

The elastic waves propagate in the direction perpendicular to the horizontal hexagonal layers, i.e., in the $z$ direction as shown in Fig. 1(a). In this direction of propagation, the theory [13] predicts the existence of two pure longitudinal modes LA and LO, two pure rotational modes $R_{1}$ and $R_{2}$ and four coupled transverse and rotational modes $\mathrm{RT}_{1}$, $\mathrm{TR}_{1}, \mathrm{RT}_{2}$ and $\mathrm{TR}_{2}$. The four coupled transverse and
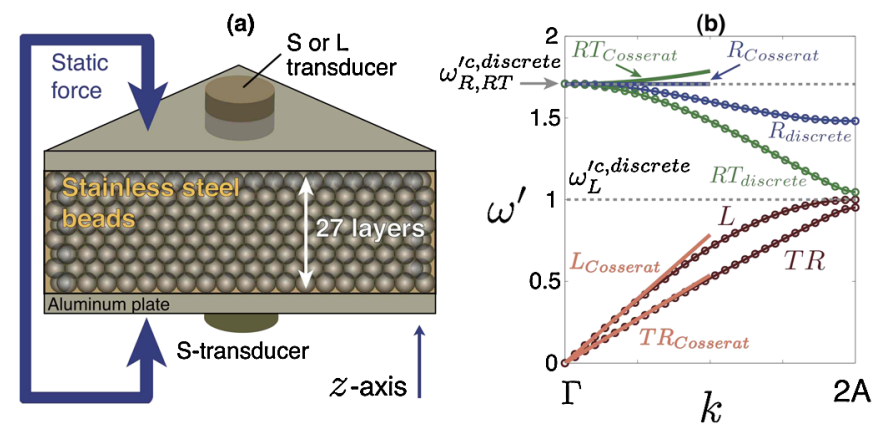

FIG. 1 (color online). (a) Experimental setup. (b) Theoretical dispersion curves for propagation in the $z$ direction of the hcp granular crystal from the discrete lattice model (circles) and their counterparts from the Cosserat continuum model (lines). The cyclic frequency is normalized to the cutoff cyclic frequency $\omega^{\prime}=\omega / \omega_{L}^{c \text {,discrete }}$ of the mode $L$. rotational modes can be split into two categories, the transverse-rotational modes (TR), which are transverse modes modified due to the presence of the rotational degrees of freedom, and the rotational-transverse modes (RT), which do not exist if the rotational degrees of freedom are not taken into account. The space group of the hcp structure contains a sixfold screw axis (or a sixfold axis of rotation-inversion) along the $z$ axis. Because of this, all mode pairs whose dispersion curves cross at the point $A=[0,0, \sqrt{3 / 8}(\pi / a)]$ of the irreducible Brillouin zone in the reciprocal space $\left(k_{x}, k_{y}, k_{z}\right)$ become degenerate at this point (the modes LO and LA for instance) $[13,25,26]$. It is therefore possible to consider the dispersion relations in an extended zone, from the point $\Gamma=(0,0,0)$ to the point $2 A$ by unfolding the dispersion curves of mode pairs as shown in Fig. 1(b). In the translational and rotational frequency ranges, the modes $L$ and RT have the highest cutoff frequencies $\omega_{L}^{c \text {,discrete }}$ and $\omega_{\mathrm{RT}}^{c \text {,discrete }}=\omega_{R}^{c \text {,discrete }}$, respectively, (the cutoff frequency of the mode RT merges with the one of the mode $R$ ). The propagative frequency bands depend on the rigidities of the contacts which in turn depend on the static normal force $N_{0}$ applied at the contact points. The cutoff cyclic frequency of mode $L$ is given by [13]

$$
\begin{aligned}
\omega_{L}^{c, \text { discrete }} & =\left\{\left[2\left(2+\Delta_{K}\right)\left(3 a N_{0}\right)^{1 / 3} E^{2 / 3}\right] /\left[m_{b}\left(1-\nu^{2}\right)^{2 / 3}\right]\right\}^{1 / 2} \\
& =\left[\left(8 K_{N}+4 K_{S}\right) / m_{b}\right]^{1 / 2},
\end{aligned}
$$

where $m_{b}$ is the mass of one bead, $E$ and $\nu$ are the Young's modulus and Poisson's ratio of the material of the beads, respectively, and $\Delta_{K}=2(1-\nu) /(2-\nu)=K_{S} / K_{N}$ is the ratio of shear $K_{S}$ to normal $K_{N}$ rigidities of the contact [10]. The cutoff frequency of the mode RT is given by $\omega_{\mathrm{RT}}^{c \text {,discrete }}=\left[10 \Delta_{K} /\left(2+\Delta_{K}\right)\right]^{1 / 2} \omega_{L}^{c \text {, discrete }}=\left[40 K_{S} / m_{b}\right]^{1 / 2}$. Observation of the dependence of the wave propagation on external loading provides an additional opportunity for testing the available theoretical predictions.

Even for macroscopically ordered structures, disorder at the microscopic level of the contacts exists caused by a weak polydispersity. In our experiments, the relative dispersion in the contact rigidities is sufficiently reduced by external loading of the granular crystal. All the measurements are repeated for five different configurations of contacts (a mechanical tap, which rearranges the contact network, is applied to the crystal) to carry out a statistical averaging of the response of the crystal. The theoretical results are compared with the experimentally measured wave transmittance through a granular crystal. The crystal is excited on one side with a shear piezo transducer as shown in Fig. 1(a). In order to discriminate the components of the transmitted acoustic waves, two different transducers are used for the detection on the other side of the crystal: a compressional transducer, sensitive only to the longitudinal mode (the mode $L$ ) and a shear transducer, preferentially sensitive to shear modes (TR and RT) but nevertheless also sensitive to the longitudinal mode (the mode $L$ ). 
Comparison of the two received signal spectra and frequency dependent group delays allows us to determine the polarization of the wave at a given frequency [8]. The acoustic measurements are carried out with different external loads corresponding to static forces applied at the contacts of 2 to $32 \mathrm{mN}$ per contact. The applied normal force $N_{0}$ is assumed to be the same for all the contacts. From these loads, the evaluated cutoff frequencies [Eq. (1)] for the mode $L$ cover the range from 60 to $97 \mathrm{kHz}$, and the evaluated cutoff frequencies of the mode RT cover the range from 103 to $166 \mathrm{kHz}$. For the estimations, we used $\nu=0.3, E=200 \mathrm{GPa}$, and $m_{b}=3 \cdot 3 \cdot 10^{-5} \mathrm{~kg}$. The typical contact diameters are of the order of $4-10 \mu \mathrm{m}$, the diameter of the beads is $2 \mathrm{~mm}$, and the wavelengths range from the bead diameter up to $10 \mathrm{~cm}$ typically. The $z$ direction is also the direction of gravity which adds to the loads at the contacts. Consequently, the cutoff frequencies are estimated twice, once at the top of the crystal with the
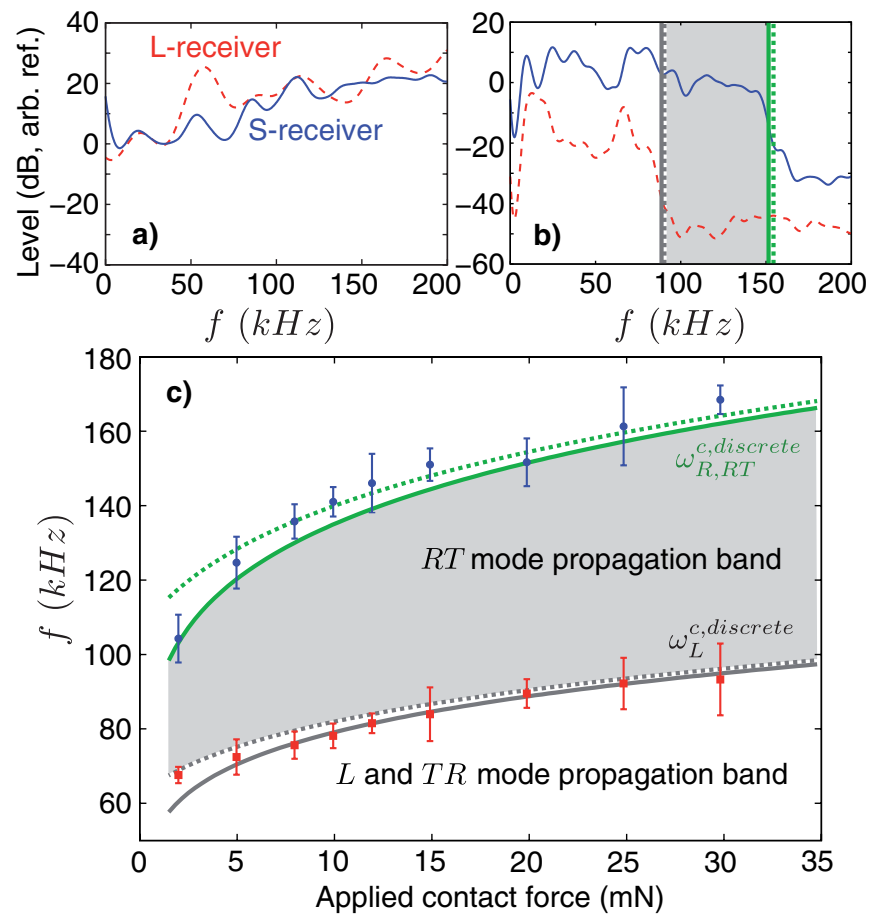

FIG. 2 (color online). Comparison of the experimental cutoff frequencies with the theoretical evaluations. (a) Wave transmittance in an aluminum reference block measured with the shear (continuous line) and compressional (dashed line) transducers. The acoustical energy is transmitted in the whole frequency range from low frequencies to $200 \mathrm{kHz}$. (b) Transmittance measured in the granular crystal under an applied static loading of $20 \mathrm{mN}$ per contact $(22 \mathrm{mN}$ at the bottom). The measurement with the shear transducer shows a cutoff frequency close to $150 \mathrm{kHz}$. The measurement with the compressional transducer shows a cutoff frequency close to $80 \mathrm{kHz}$. (c) Dependence of the cutoff frequencies measured with the shear (circles) and compressional (squares) transducers on the external loading. Error bars correspond to a $90 \%$ confidence interval. static loading only and the second time at the bottom with the weight of the crystal added to the static loading. The theoretically evaluated cutoff frequencies reported in Fig. 2 are in agreement with the experimental measurements. When the receiving transducer is sensitive only to compression (mode $L$ ), the results show transmission cutoff frequencies between 60 and $95 \mathrm{kHz}$, which correspond to the cutoff frequencies of mode $L$. When the receiving transducer is sensitive to both longitudinal and shear displacements (modes $L$ and RT), the measured cutoff frequencies are situated between 105 and $170 \mathrm{kHz}$, which correspond to the cutoff frequencies of mode RT. The frequency range of propagation of the RT mode lies between the cutoff frequencies observed with the compressional transducer and those observed with the shear transducer. The good agreement between the theoretical and measured variations of the cutoff frequencies with the external static loading confirms the good description given by the theory.

Exciting the system with a short pulse of $11 \mu$ s duration, all acoustic frequencies are emitted nearly simultaneously. In this case, the dependence of the wave arrival time on frequency provides information on the dispersion relations of the different modes. The theoretical arrival times are predicted using the group velocities deduced from the dispersion relations. In Fig. 3, the spectrograms of the transmitted signals measured with the compressional and the shear transducers are presented. With the compressional receiving transducer, only the frequencies corresponding to mode $L$ are visible, and the measured arrival times correspond to those calculated from theory. With the shear receiving transducer, the frequencies corresponding to the mode RT are also visible, and the arrival times correspond to the theoretical curves of the modes $L$, TR,

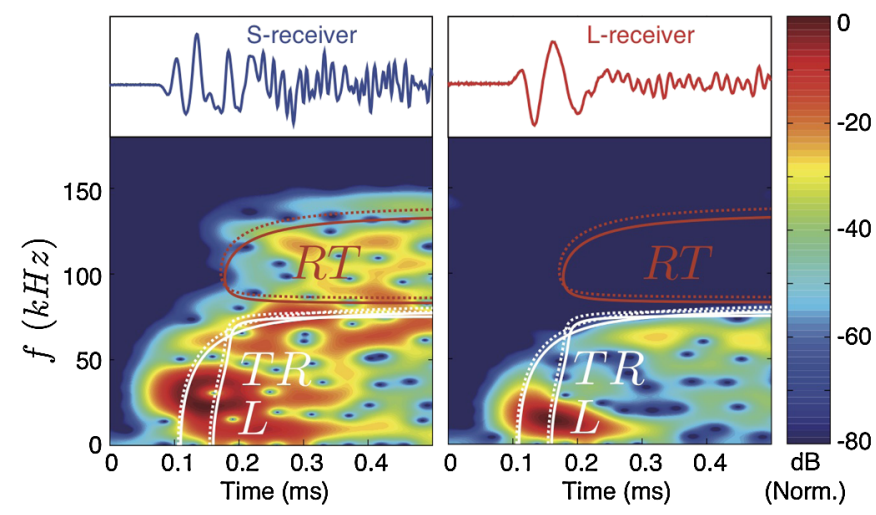

FIG. 3 (color online). Received signals from a shear transducer (left) and a longitudinal transducer (right) after transmission through a crystal under static loading of $10 \mathrm{mN}$ per contact (12 $\mathrm{mN}$ at the bottom). The excited shear pulse is centered on $200 \mathrm{kHz}$. Normalized temporal signals are shown at the top, the spectrograms at the bottom. Theoretical arrival times of the modes are shown in continuous lines $(10 \mathrm{mN}$ per contact) and dashed lines (12 $\mathrm{mN}$ per contact). 
and RT. The experimental arrival times of modes $L$, TR, and RT look similar to the theoretical predictions, although the spectrogram tool has some intrinsic uncertainty both in time and frequency determination [27]. The good agreement between theory and experiment shows convincing evidence that the RT mode is experimentally observed.

The experimental observation above, indicating wave propagation in granular crystals that can only exist in the presence of bead rotation interactions, is a direct confirmation of the Cosserat idea that the rotational degrees of freedom should be, in general, taken into account in the description of the elastic behavior of micro-inhomogeneous materials. It is, then, interesting to understand how useful the Cosserat theory could be for the description of long-wavelength elastic waves in granular crystals when the medium properties can be homogenized and the medium is considered isotropic in a first approximation. We should compare the granular crystal in the limit $k_{z} \rightarrow 0$ with the Cosserat continuum characterized by a single nonzero nonclassical elastic constant $\alpha$ [8]. This constant introduces asymmetry of the ordinary stress tensor which is caused by the existence of the density of the moment of inertia $J$. The latter can be found in our hexagonal close-packed granular crystal precisely due to the knowledge of the packing density $\eta=\pi /(3 \sqrt{2}) \simeq$ 0.74 and the moment of inertia of an individual bead $I=(1 / 10) m_{b} a^{2}$. From the values of the packing density, it follows that there are $\sqrt{2}$ beads in a cube with an edge equal to $a$. Thus, it provides the estimate $J=\sqrt{2} I / a^{3}=$ $\eta \rho_{b} a^{2} / 10$ where $\rho_{b}$ is the density of the material constituting the beads. At the next step, the nonclassical constant $\alpha$ is estimated from the comparison of our theoretical prediction for the common cutoff frequency $\omega_{R, \mathrm{RT}}^{c \text {,discete }}$ of the $R$ and RT modes with the theoretical value for the same frequency in the Cosserat continuum [4] $\omega_{R, \mathrm{RT}}^{c, \text { Cosserat }}=2(\alpha / J)^{1 / 2}$. We find $\alpha=\sqrt{2} K_{S} / a$. From the comparison of the velocities in the limit of infinitely low frequencies of the TR modes in the granular crystal $v_{\mathrm{TR}}^{\text {discrete }}=\left[a^{2}\left(K_{N}+2 K_{S}\right) /\left(3 m_{b}\right)\right]^{1 / 2}$ and in the Cosserat continuum $v_{\mathrm{TR}}^{\text {Cosserat }}=(\mu / \rho)^{1 / 2}$, where $\rho=\eta \rho_{b}$ is the density of the homogenized media, the shear rigidity of the homogenized material $\mu=\sqrt{2}\left(K_{N}+2 K_{S}\right) /(3 a)$ is obtained. The ratio $\alpha / \mu=(6-6 \nu) /(6-5 \nu)$ characterizing the asymmetry of the stress tensor is about 0.93 for the beads with the Poisson's ratio $\nu \simeq 0.3$.

However, with the defined parameters of the hypothetical Cosserat continuum, the Cosserat theory is unable to reproduce the dispersion relations for the long waves in the noncohesive granular crystal, i.e., at small but finite wave numbers $\left(k_{z} \rightarrow 0, k_{z} \neq 0\right)$ as shown in Fig. 1(b). In the Cosserat continuum, the purely rotational mode $R$ becomes dispersionless, while the dispersion curve of the RT mode, which emerges from the frequency $\omega_{R, \mathrm{RT}}^{c \text {, Cosserat }}$ common with the $R$ mode, is concave upward due to the repulsion of the rotation-dominated and shear-dominated (RT and TR, respectively) acoustic modes. In fact, the positive concavity is a rather general feature of the Cosserat continuum, even in the presence of the moment stresses or in the anisotropic Cosserat continuum [28], and in such generalizations of the Cosserat continuum as microstretch continuum and micromorphic continuum [2]. Contrary to this, the dispersion curves of the $R$ and RT modes, which emerge in the discrete model from the common $\omega_{R, \mathrm{RT}}^{c \text {,discete }}$, are concave downward in the hexagonal and cubic noncohesive granular crystals $[13,15]$ as shown in Fig. 1(b).

To understand the reasons for these differences between the discrete and the Cosserat theory, it is insightful to consider the limiting case $J / \rho \rightarrow 0$, by assuming that the density $J$ of the moment of inertia tends to zero and keeping the same density $\rho$ of the medium. This formally represents the situation where the mass of an individual bead is $\delta$ localized at the center of the sphere. The analysis demonstrates that the propagating frequency bands of the dispersion relations of the two rotational modes tend to infinity [13], while the frequency bands of the propagating translational modes remain finite. This cancels the effect of mode repulsion on the bending of the dispersion relations. In the Cosserat continuum, mode RT becomes dispersionless while both rotational modes in the discrete theory are concave downward. This demonstrates the dominance of material periodicity (inhomogeneity), which induces coherent wave scattering, over mode repulsion and explains the concave downward dispersion relations of real granular crystals shown in Fig. 1(b) (i.e., where $J / \rho$ is nonzero). Though the analysis indicates that positive concavity of the dispersion curves for the $R$ and RT modes in threedimensional granular crystals could be caused by strong bending rigidity $[9,15,29]$, this does not change the following general conclusion derived from the comparison of wave propagation in granular crystals to that in the Cosserat continuum. The spatial inhomogeneity of the materials strongly influences the dispersion relations of the eigenmodes by inducing wave scattering and the additional rotational degrees of freedom. The Cosserat theory accounts for the possible rotations and formally incorporates a characteristic scale of inhomogeneity. However, the Cosserat theory describes the possible wave scattering phenomena in microinhomogeneous media at least incompletely. The generalized elasticity theory valid for the description of long waves in granular crystals should also explicitly incorporate the spatial scale of inhomogeneity in order to account for the multiple scattering of the waves. Thus, the Cosserat theory should be combined with higher gradient theories [29-31]. It can be verified that the continuum models for the granular media [29,31], which have been derived by homogenization of the discrete equations of motion for isotropic disordered granular packings, predict the negative concavity of the dispersion curves for the $R$ and RT modes when the couple stresses are negligible. 
In summary, the influence on elastic wave propagation of interactions related to rotational degrees of freedom in noncohesive granular crystal has been studied. The propagating rotational-transverse mode existing due to the rotational degrees of freedom has been revealed experimentally. Theoretical comparison of the waves in the homogenized granular crystals with those in the Cosserat continuum has demonstrated that the Cosserat theory does not account for all the influences of the material inhomogeneity on its elastic behavior.

The authors would like to like to acknowledge Laurent Simon for fruitful discussions. This work has been supported by ANR Project STABINGRAM No. ANR-2010BLAN-0927-03.

*aurelien.merkel.etu@univ-lemans.fr

[1] E. Cosserat and F. Cosserat, Théorie des Corps Déformables (Hermann et Fils, Paris, 1909).

[2] A. Eringen, Microcontinuum Field Theories. I. Foundations and Solids (Springer-Verlag, New York, 1999).

[3] R. Lakes, in Continuum Models for Materials with Microstructure, edited by H. Muhlhaus (John Wiley and Sons, New York, 1995).

[4] W. Nowacki, Theory of Asymmetric Elasticity (Pergamon, Oxford, 1986).

[5] E. Pasternak and A. Dyskin, in Mechanics of Generalized Continua: One Hundred Years After the Cosserat, edited by G. A. Maugin and A. V. Metrikine (Springer-Verlag, New York, 2010).

[6] A. Kiris and E. Inan, Int. J. Eng. Sci. 46, 585 (2008).

[7] R.D. Gauthier, in Mechanics of Micropolar Media, edited by O. Brulin and R. K. T. Hsieh (World Scientic Publishing, Singapore, 1982).

[8] See Suplemental Material at http://link.aps.org/ supplemental/10.1103/PhysRevLett.107.225502 for details on the Cosserat and discrete models, experimental setup and a note on the bibliography.

[9] I. Pavlov, A. I. Potapov, and G. A. Maugin, Int. J. Solids Struct. 43, 6194 (2006).

[10] J. Duffy and R. D. Mindlin, J. Appl. Mech. 24, 585 (1957).

[11] V.F. Nesterenko, Dynamics of Heterogeneous Materials (Springer-Verlag, New York, 2001).

[12] L. M. Schwartz, D. L. Johnson, and S. Feng, Phys. Rev. Lett. 52, 831 (1984).

[13] A. Merkel, V. Tournat, and V. Gusev, Phys. Rev. E 82, 031305 (2010).

[14] K.L. Johnson, Contacts Mechanics (Cambridge University Press, Cambridge, 1985).

[15] A. S. J. Suiker, A. V. Metrikine, and R. de Borst, Int. J. Solids Struct. 38, 1563 (2001).

[16] Z. Liu et al., Science 289, 1734 (2000).

[17] Z. Liu, C. T. Chan, P. Sheng, A.L. Goertzen, and J. H. Page, Phys. Rev. B 62, 2446 (2000).

[18] S. Yang et al., Phys. Rev. Lett. 88, 104301 (2002).

[19] W. Cheng et al., Nature Mater. 5, 830 (2006).

[20] T. Still et al., Phys. Rev. Lett. 100, 194301 (2008).

[21] A. V. Akimov et al., Phys. Rev. Lett. 101, 033902 (2008).

[22] M. de Billy, J. Acoust. Soc. Am. 110, 710 (2001).

[23] J. Anfosso and V. Gibiat, Europhys. Lett. 67, 376 (2004).

[24] J. H. Page et al., Phys. Status Solidi (b) 241, 3454 (2004).

[25] L. J. Raubenheimer and G. Gilat, Phys. Rev. 157, 586 (1967).

[26] M. T. Dove, Introduction to Lattice Dynamics (Cambridge University Press, Cambridge, 1993).

[27] P. Flandrin, Time-Frequency/Time-Scale Analysis (Academic Press, San Diego, London, 1999).

[28] S. Minagawa, K.-I. Arakawa, and M. Yamada, Bull. JSME 24, 22 (1981).

[29] A. S. J. Suiker, R. de Borst, and C. S. Chang, Acta Mech. 149, 161 (2001); 149, 181 (2001).

[30] C. S. Chang and J. Giao, Int. J. Non-Linear Mech. 30, 111 (1995).

[31] H.-B. Mühlhaus and F. Oka, Int. J. Solids Struct. 33, 2841 (1996). 